\title{
Stimulus-specific processing consequences of pattern goodness
}

\author{
MARC M. SEBRECHTS and W. R. GARNER \\ Yale University, New Haven, Connecticut 06520
}

\begin{abstract}
Two issues concerning the effects of visual pattern goodness on information processing time were investigated: the role of memory vs. encoding and the role of individual stimulus goodness vs. stimulus similarity. A sequential "same-different" task was used to provide differentiation of target item or memory effects from display item or encoding effects. Experiment 1 used four alternative stimuli in each block of trials. The results showed that good patterns were processed faster than poor patterns for both "same" and "different" responses. Furthermore, the goodness of the target item had a greater effect on reaction time than did the goodness of the display item, indicating that memory is more important than encoding in producing faster processing of good stimuli. Effects of interstimulus similarity on processing time were minimal, although isolation of good stimuli in a similarity space could explain many of the results. Experiment 2 replicated the results of Experiment 1, despite the fact that differences in similarity space had been minimized by using only two alternative stimuli in each block. In addition, the speed of processing a "same" pair was essentially independent of the particular alternative stimulus in a block. These results suggest that in this task, there is a processing advantage for good stimuli that is stimulus specific, with the effect operating primarily in memory.
\end{abstract}

It is widely recognized that the structure of visual objects affects phenomenal experience, although the precise nature of that effect is not fully known. The Gestalt psychologists made substantial progress in understanding these experiences by enumerating characteristics of patterns that made them perceptually "good." More recently, Garner (1962) indicated how such goodness might be related to stimulus information and redundancy. He suggested that for a given pattern, the number of equivalent patterns provided a useful index of what constitutes a good figure: The fewer the number of equivalent patterns, the better the given pattern will be.

For experimental purposes, rotations and reflections serve as one useful specification of equivalent patterns. If rotating and/or reflecting a pattern yields the same pattern, the number of equivalent patterns is one and the pattern is good; if rotating or reflecting a pattern yields several different patterns, the number of equivalent patterns is larger and the pattern is poorer. Garner and Clement (1963) showed that this definition was highly correlated with the way people rate goodness, thus providing a well-defined means of specifying a good or a poor pattern independent of subjective ratings.

\section{Processing Consequences of Goodness}

Using a variety of information processing tasks, it has been found that good patterns are processed more efficiently than poor patterns, as measured by time or

This research was supported by Grant MH14229 from the National Institute of Mental Health to Yale University. Requests for reprints should be sent to either author, Department of Psychology, Box 11-A Yale Station, New Haven, Connecticut 06520. by errors. Since it has been shown that there exists a reliable processing advantage for good patterns, recent research has been primarily concerned with establishing the nature of that effect. In particular, this research has tried to determine whether the better processing of good patterns is due to encoding or memory factors.

Encoding effects of goodness. A series of recent studies has argued for encoding effects of figural goodness. Clement and Varnadoe (1967) conducted a twochoice speeded classification task in which subjects sorted decks of cards with two alternative dot patterns per deck. The alternatives were both good, both poor, and one good and one poor. The decks with two good patterns were classified most rapidly, the decks with two poor patterns were classified most slowly, and the decks containing one good and one poor pattern yielded intermediate results. Clement and Varnadoe argued that their results were due to a faster encoding of the good patterns.

The card-sorting technique did not allow determination of reaction times (RTs) to individual patterns of the pair to be discriminated, so Garner and Sutliff (1974) carried out the equivalent experiment with a discrete choice reaction procedure. One particular stimulus was assigned to each of two response keys. Stimuli appeared one at a time, and the subject was required to indicate which of the two stimuli had appeared. There were three conditions: two good stimuli, two poor stimuli, and one good stimulus and one poor stimulus. The conditions with one or two good stimuli were both faster than that with two poor stimuli. In addition, reactions to good stimuli within the good-poor condition were faster than reactions to poor stimuli in that condi- 
tion. This difference was attributed to an encoding stage without specifying precisely the nature of that stage.

Ruth (1976) used a similar two-choice reaction procedure to extend these results through an examination of the effects of expectancy on RT to good and poor patterns. Expectancy was specified by cues indicating the probability of occurrence of particular stimuli or goodness levels. She found that reaction to both expected and unexpected good targets was quicker than reaction to expected and unexpected poor targets, respectively. Ruth assumed that the expected items are held in memory. She argued that an unexpected item does not affect memory and a faster response for unexpected good targets therefore indicates an effect of goodness on encoding.

An encoding effect of goodness was also supported by Bell and Handel (1976). They showed that under backward masking, good patterns were more accurately reproduced than poor ones, although there was no such difference in the absence of the mask. Good patterns, they argued, were more fully encoded before the onset of the mask.

Memory effects of goodness. Checkosky and Whitlock (1973) utilized the Sternberg memory-scanning task to address similar questions. Two or three patterns of a particular goodness level were presented as memory items. A test item was displayed to the subject, who was then required to indicate as rapidly as possible whether or not the item was in the memory set. The investigators found that for positive responses, there were no changes in the intercepts of functions with items from different goodness levels, although there were slope differences. Following the stage analysis of Sternberg (1967), Checkosky and Whitlock suggested that goodness had influenced memorial comparison rather than encoding. After additional analysis of these data, Garner (1974) noted that in the case of negative responses, there is a change in intercept for test items of different goodness. Using the same logic that had been followed by Checkosky and Whitlock, Garner concluded that there is also an encoding effect of goodness.

Pomerantz (1977) pointed out that the Checkosky and Whitlock (1973) study was restricted by certain assumptions of the additive-factors approach. In addition, the two-choice reaction tasks had not controlled what item or items were being held in memory, since the subject was free to use a number of different memory strategies. Pomerantz tried to clarify the issue, using a two-choice reaction procedure, by assigning a single stimulus to one response and two or four stimuli to the second response. His assumption in using this procedure was that the subject would "focus" on the single stimulus that was assigned its own response and would thus hold in memory only that item and not the larger number of items assigned to the second response. The subject would then presumably use a go/no-go strategy of simply deciding whether or not the presented stimulus was the single item in memory. Pomerantz' results showed that only the goodness of the focused stimulus affected RT (good stimuli again being faster); from this result, Pomerantz concluded that goodness had its effect on memory, not on encoding, in this task.

\section{Goodness and Similarity Effects}

In the various information processing tasks used in this research, goodness of individual stimuli clearly affects RT. The effects thus appear to be stimulus specific. However, in any of these tasks, two or more stimuli are actually used, and these stimuli have similarity relations in addition to the stimulus-specific property of goodness. In order to clarify the effects of goodness as distinct from the effects of similarity, it is necessary to distinguish between two types of similarity.

Interstimulus similarity. Between the members of any pair of stimuli, there is a similarity, and in any task involving differentiation between two stimuli, this similarity may have an effect on RT quite independently of the effects of goodness. For example, Gamer and Sutliff (1974), in using a two-stimuli choice RT procedure, found that the interstimulus similarity of a pair of stimuli did affect RT. The effects of goodness in this and in similar tasks, however, are demonstrated by showing that particular stimuli with different goodness have different RTs even though they share a common interstimulus similarity. In the Garner and Sutliff example, good stimuli were responded to faster than poor stimuli when paired against each other. Thus goodness effects were shown in addition to similarity effects.

Similarity space. If more than two stimuli are used in a particular task, then there still exist interstimulus similarities for all of the possible pairs, and these might well influence RT, much as they do when only two stimuli are used in a task. In addition, however, there is an overall similarity space involving similarity relations between all stimuli in the total set. This similarity space can have additional effects on particular stimuli (Lockhead, 1972), because each stimulus has a unique relation to the entire set of stimuli. For example, stimuli may differ in how close the nearest other stimulus is or in how close on the average each stimulus is to all the others.

The concept of similarity space relates to the present research in two ways. First, good stimuli are more isolated in a similarity space. Garner $(1962,1974)$ has emphasized that good patterns have few alternative or equivalent patterns; thus they should be isolated in a similarity space. Lockhead and King (1977) have indeed shown with similarity ratings of some stimuli for which the concept of goodness is meaningful that good patterns are more isolated than poor patterns. More recently, King, Crist, and Lockhead (1979) demonstrated that under certain conditions similarity relations can predict RT for such patterns. 
The second reason for interest in the idea of a similarity space is that its role need not be the same for encoding and for memory functions. Thus an investigation of the roles of memory and encoding should aid in understanding the role of similarity space in the processing of pattern goodness.

\section{Purpose of this Research}

We conducted two experiments designed to clarify further the role of goodness in information processing by using a task that would allow better separation of the roles of memory and encoding in influencing the nature and magnitude of any effects of goodness. In Experiment 1, we obtained RT data for a set of four alternative stimuli; we also collected both goodness and similarity ratings to allow a comparison of the respective roles of these two factors. In Experiment 2, we used just two alternative stimuli in any single experimental block, in order to eliminate any effects due to a similarity space. In addition, by using different alternative stimuli in different blocks, with different interstimulus similarities, we could determine more directly the nature of goodness effects over and above any similarity effects.

\section{GENERAL METHOD}

The task we chose was the sequential "same-different" task, because it provides a clear separation between the functions of the two stimuli: The first stimulus presented, the target, is to be held in memory and compared with the second stimulus presented, the display. The response is then to indicate whether or not the second item is the same as that held in memory. Both the first and second items have to be encoded, of course, but, with a reasonable delay between the target and display item, it can be assumed that encoding of the first item is completed before presentation of the second item. Thus independent manipulations of the goodness of the first and second stimuli will allow specification of the relative importance of memory and encoding effects.

\section{Stimuli}

The stimuli used in this paper are the four patterns of dots shown in Figure 1; they are identical to four of the patterns used by Garner and Sutliff (1974). Each of these patterns was generated by placing nine dots in the cells of an imaginary 5 by 5 matrix, with at least one dot in each column and each row. Each pattern is labeled with a digit and a letter. The digit indicates the number of different patterns that result from all possible rotations in steps of $90 \mathrm{deg}$ and/or reflections about vertical, horizontal, or diagonal axes of the given pattern (the $R \& R$ subset size); the letter merely differentiates specific stimuli belonging to a certain subset size. The patterns represent two different goodness levels by the R\&R criteria. Two of the patterns (1a and 1b) are generated from subsets of Size 1, and two ( $8 a$ and $8 b$ ) from subsets of Size 8 (see Garner, 1974, Lecture 1). For these stimuli, symmetry provides a similar distinction. Patterns $1 \mathrm{a}$ and $1 \mathrm{~b}$ have vertical, horizontal, and diagonal symmetry; $8 \mathrm{a}$ and $8 \mathrm{~b}$ have no symmetry about a major axis.

The patterns were printed on slides and were back-projected onto a screen, with the patterns showing as black dots against a white background. The subjects viewed the patterns in a sounddeadened room ( $2.13 \mathrm{~m}$ square), with a constant low-intensity illumination.

\section{Conditions}

In an attempt to extract the relevant import of first and second items in this paradigm, we will consider three distinct types of pairings or conditions and their respective subconditions.

Same stimuli. The first condition consists of those stimulus pairs that are composed of identical target and display items and require a "same" response. A subcondition consists of "same" pairs from the same goodness level. Thus la1a and $1 \mathrm{~b} 1 \mathrm{~b}$ would belong to one subcondition, and $8 \mathrm{a} 8 \mathrm{a}$ and $8 \mathrm{~b} 8 \mathrm{~b}$ would belong to a different subcondition.

a b

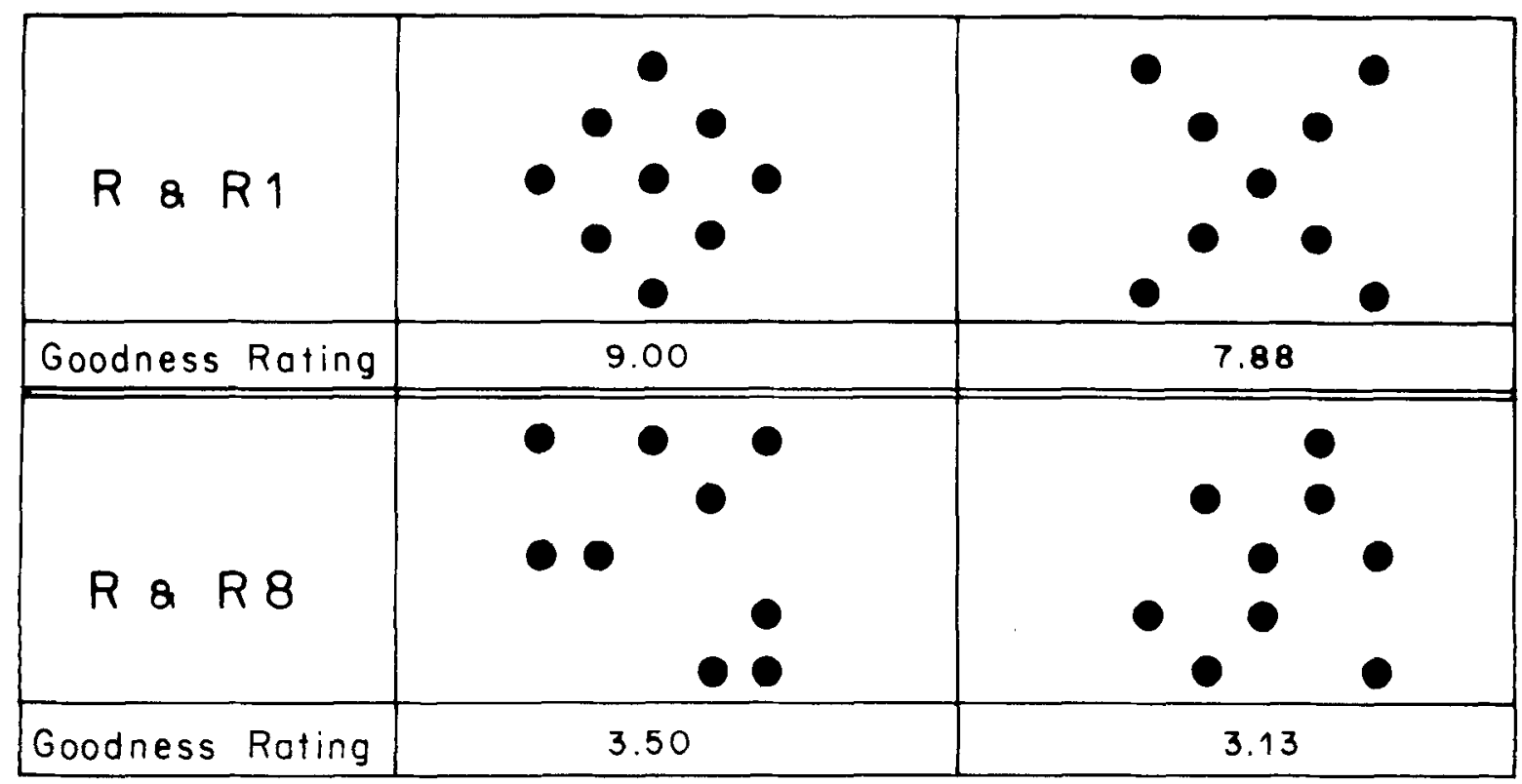

Figure 1. The four patterns used in Experiments 1 and 2 and their associated mean goodness ratings as obtained in Experiment 1 on a 10-point scale. The stimuli are identified in the text by a number indicating $R \& R$ subset size and a letter indicating the specific stimulus of a particular $R \& R$ subset size. 
Different stimuli, same goodness level. The second stimulus condition is made up of stimulus pairs that have different stimuli but come from the same goodness level and require a "different" response. A subcondition consists of all pairs belonging to a particular goodness level. Thus $1 \mathrm{alb}$ and $1 \mathrm{bla}$ would belong to one subcondition, and $8 \mathrm{a} 8 \mathrm{~b}$ and $8 \mathrm{~b} 8 \mathrm{a}$ would belong to a different subcondition.

Different stimuli, different goodness levels. The third stimulus condition includes the remaining pairs that require the "different" response; these pairs consist of stimuli from different goodness levels. The subconditions consist of pairs for which the goodness level of the target and display are in the same order. Thus $1 \mathrm{a} 8 \mathrm{a}, 1 \mathrm{a} 8 \mathrm{~b}, 1 \mathrm{~b} 8 \mathrm{a}, 1 \mathrm{~b} 8 \mathrm{~b}$ would belong to one subcondition, and $8 \mathrm{a} 1 \mathrm{a}, 8 \mathrm{a} 1 \mathrm{~b}, 8 \mathrm{~b} 1 \mathrm{a}, 8 \mathrm{~b} 1 \mathrm{~b}$ would belong to a different subcondition. For each stimulus pair in one subcondition, there is a pair with the same stimuli in a reversed order in the other subcondition.

\section{EXPERIMENT 1: FOUR STIMULUS ALTERNATIVES}

\section{Method}

Subjects. Eight right-handed Yale graduate students, four male and four female, were paid to serve as subjects for a total of approximately $5 \mathrm{~h}$ each.

Stimuli. The four patterns in Figure 1 were used. The projected dots were $2 \mathrm{~mm}$ in diameter, with the centers of adjacent dot locations being separated by $8 \mathrm{~mm}$ horizontally and vertically. The subjects viewed the patterns at a distance of approximately $1.0 \mathrm{~m}$. The total pattern thus subtended a maximum vertical or horizontal visual angle of approximately $2.0 \mathrm{deg}$, and both target and display stimuli were presented in the center of the viewing field.

Reaction time procedure. The task required a sequential "same-different" identification. On each trial, one of the four stimuli was presented as the first (target) item for $1.0 \mathrm{sec}$, followed by a $1.0-\mathrm{sec}$ delay. One of the same four stimuli was then presented as the second (display) item; it remained on until a response was made. There was a delay of $4.75 \mathrm{sec}$ after the end of one trial to the beginning of the next, during which the experimenter recorded the response and the RT to the display stimulus and changed slides. RT was measured by an electronic clock to the nearest millisecond. Timing and measurement were controlled by logic circuitry.

Responses were made by tapping one of two response keys located $12 \mathrm{~cm}$ apart. All subjects used their right hands to depress the right key and their left hands to depress the left key. Four subjects (two male and two female) were verbally instructed to press the right-hand key if the stimuli were the same and the left-hand key if the stimuli were different; the remaining four subjects were given the reverse response assignment.

At the beginning of the experiment, the subject was shown exemplars of the four stimuli without details of timing or sequence. The subject was instructed to respond as rapidly as possible without making errors.

Experimental design. Each subject participated in three sessions on 3 consecutive days. Each session lasted approximately $1 \mathrm{~h}$ and $40 \mathrm{~min}$ and consisted of 24 practice trials followed by six blocks of 96 trials each. The subject was given a short break after each block. The subject was informed that the first set of trials was practice or warm-up.

On each block of 96 trials, half were same pairs ( 12 trials of each of the 4 pairs of same stimuli), and half were different pairs (4 trials of each of the 12 pairs of different stimuli). Thus each response was required for half of the trials. The pairs of stimuli were presented in quasirandom order, with the following constraints: No memory or display item could occur in more than three successive trials, and neither response could occur more than three times consecutively.

Analyses. Each session provided for each subject a total of
72 trials for each of the same pairs and 24 trials for each of the different pairs. Error trials were eliminated, and a median RT was calculated for each subject for each of the 16 stimulus pairs for each of the three sessions. These medians provided the basis for all further RT analyses. Statements of significance are at $p<.05$ and are based on analyses of variance with NewmanKeuls tests for pairwise comparisons.

Mean error rates for each session were $2 \%$, and there was no indication of a speed-accuracy tradeoff. No further analyses of errors were made.

Rating procedure. At the end of the RT portion of the experiment, two types of ratings were obtained from the subjects: (1) The goodness of each of the four stimuli was rated on a scale of 1 to 10 , with 1 being very poor and 10 being very good; (2) the similarity between the six nonidentical pairs of stimuli was also rated on a scale of 1 to 10, with higher numbers meaning more similar. For both of these ratings, the stimuli were shown on cards placed in a fixed 2 by 2 order; only a single rating was obtained for each stimulus or stimulus pair, with no counterbalancing for position.

\section{Results and Discussion}

The goodness ratings obtained for the four patterns are indicated under each pattern in Figure 1. These ratings simply confirm that the patterns from the two different $R \& R$ subset sizes do differ substantially in rated goodness in the expected direction.

A preliminary analysis of variance of the RT data (sex by hand by subject by session by condition) estab. lished that there were no main effects of either sex or hand and no significant interactions involving these factors. Therefore, all subsequent analyses used data collapsed across sex and hand. While there was a significant decrease in overall RT from the first to the third session (means per session were 503, 444, and $423 \mathrm{msec}$ ), this change did not significantly interact with any of the principal comparisons. Therefore, the mean values for the various conditions are presented in Table 1 , collapsed across sessions.

Effects of conditions. Overall mean RT to same trials was faster than that for different trials. Since the relative speed of the two types of response is easily influenced by the number of trials of each type, no specific interpretation is given to this difference. Furthermore, this difference is not directly relevant to the issues being addressed in this experiment.

Same stimuli. Mean RT for each of the four stimulus pairs requiring a "same" response is shown in the first portion of Table 1. The two poor-poor pairs had virtually identical mean RTs. The good-good pairs differed by $19 \mathrm{msec}$, which is not statistically significant. However, the mean difference between the good-good pairs and the poor-poor pairs was a significant $48 \mathrm{msec}$. Thus, while there were no differences in RT within a subcondition, the good-good subcondition was considerably faster than the poor-poor subcondition.

Different stimuli, same goodness level. As shown in the middle of Table 1, four of the stimulus pairs that require a "different" response are composed of a target and a display item from a single goodness level. Within each subcondition (good-good and poor-poor), 
Table 1

Mean Reaction Time (in Milliseconds) for Each Stimulus Pairing in Experiment 1 : Four Stimulus Alternatives

\begin{tabular}{|c|c|c|c|c|c|c|c|c|c|c|c|}
\hline & & & & \multicolumn{8}{|c|}{ Different Stimuli } \\
\hline \multicolumn{4}{|c|}{ Same Stimuli } & \multicolumn{4}{|c|}{ Same Goodness Level } & \multicolumn{4}{|c|}{ Different Goodness Levels } \\
\hline \multicolumn{2}{|c|}{ Good-Good } & \multicolumn{2}{|c|}{ Poor-Poor } & \multicolumn{2}{|c|}{ Good-Good } & \multicolumn{2}{|c|}{ Poor-Poor } & \multicolumn{2}{|c|}{ Good-Poor } & \multicolumn{2}{|c|}{ Poor-Good } \\
\hline $\begin{array}{l}\text { lala } \\
\text { lblb }\end{array}$ & $\begin{array}{l}389 \\
408\end{array}$ & $\begin{array}{l}8 \mathrm{a} 8 \mathrm{a} \\
8 \mathrm{~b} 8 \mathrm{~b}\end{array}$ & $\begin{array}{l}446 \\
446\end{array}$ & $\begin{array}{l}1 \mathrm{alb} \\
1 \mathrm{~b} 1 \mathrm{a}\end{array}$ & $\begin{array}{l}451 \\
449\end{array}$ & $\begin{array}{l}8 a 8 b \\
8 b 8 a\end{array}$ & $\begin{array}{l}477 \\
475\end{array}$ & $\begin{array}{l}1 \mathrm{a} 8 \mathrm{a} \\
1 \mathrm{a} 8 \mathrm{~b} \\
1 \mathrm{~b} 8 \mathrm{a} \\
1 \mathrm{~b} 8 \mathrm{~b}\end{array}$ & $\begin{array}{l}460 \\
482 \\
452 \\
459\end{array}$ & $\begin{array}{l}8 \mathrm{a} 1 \mathrm{a} \\
8 \mathrm{~b} 1 \mathrm{a} \\
8 \mathrm{alb} \\
8 \mathrm{~b} 1 \mathrm{~b}\end{array}$ & $\begin{array}{l}465 \\
489 \\
484 \\
477\end{array}$ \\
\hline Mean & 398 & & 446 & & 450 & & 476 & & 463 & & 479 \\
\hline
\end{tabular}

Note-Means for each pairing are collapsed across blocks, sessions, and subjects.

the two pairs had the same two stimuli but in the reversed order. Both of the two good-good pairs were faster than both of the two poor-poor pairs, with a significant mean difference of $26 \mathrm{msec}$. In contrast, within a goodness level, the difference was a nonsignificant $2 \mathrm{msec}$ for both good-good and poor-poor pairs. Thus the order of stimuli within a pair does not affect RT, although the goodness level of the pair does.

These results show that, once again, good patterns are processed faster than poor patterns, that the effect is not due solely to the particular order in which a pair of stimuli is presented, and that the effect occurs both when a "different" response is required and when a "same" response is required.

Different stimuli, different goodness levels. There were eight stimulus pairs that contained one good and one poor pattern, as shown in the last portion of Table 1 . Four pairs had a good pattern as target and a poor pattern as display, and four pairs had these stimuli in reversed order. For each pair of stimuli, RT was shorter when the good stimulus was the target item, with differences ranging from $5 \mathrm{msec}$ for Stimuli $1 \mathrm{a}$ and $8 \mathrm{a}$ to $32 \mathrm{msec}$ for Stimuli $1 \mathrm{~b}$ and $8 \mathrm{a}$. For each ordered pair, the difference did not reach statistical significance, but for all four pairs together, the mean difference of $16 \mathrm{msec}$ was significant.

These results show that the effect of goodness on processing is not restricted to pairs in which the goodness level of the two stimuli is the same. Furthermore, they indicate that the faster processing occurs when the target item is good. In isolation, this result is not fully interpretable, since when good stimuli are the targets, poor stimuli are the displays. Strictly speaking, it is possible to state the results as follows: Better performance occurs when poor stimuli are in the display. This way of stating the result, however, is inconsistent with data from the other two conditions. For both "same stimuli" and "different stimuli, same goodness level" conditions, good stimuli were processed faster than poor stimuli. Additional analyses will attempt to clarify this interpretation.

Effects of target and display. Two comparisons of conditions involved varying goodness of target items while holding goodness of the display item constant: good-good vs. poor-good and good-poor vs. poor-poor. All of the good-good pairs were faster than all of the poor-good pairs, with a significant mean difference of $29 \mathrm{msec}$. Six of the eight possible individual comparisons showed good-poor pairs to be faster than poor-poor pairs, with a significant mean difference of $13 \mathrm{msec}$. Thus with the goodness of the display item held constant, good target patterns produced faster processing than poor target patterns.

Two other comparisons of conditions held goodness of target item constant while varying the goodness of the display item: good-good vs. good-poor and poor-poor vs. poor-good. The effect of display item goodness depended on the goodness level of the target item. With a poor item as target, the goodness level of the display item had virtually no effect on speed of processing ( $3 \mathrm{msec}$ ), but with a good item as target, a good display item was processed faster (a significant $13 \mathrm{msec}$ ) than a poor display item.

Effects of similarity. The obtained similarity ratings for the six pairs of stimuli were $8 \mathrm{a} 8 \mathrm{~b}=7.75,1 \mathrm{a} 1 \mathrm{~b}=$ $4.75,1 \mathrm{a} 8 \mathrm{~b}=3.81,1 \mathrm{~b} 8 \mathrm{a}=3.81,1 \mathrm{a} 8 \mathrm{a}=3.38,1 \mathrm{~b} 8 \mathrm{~b}=$ 3.31. Briefly, the two poor patterns were most similar, the two good patterns were next most similar, and the greatest dissimilarity was between the good and the poor patterns, with an average similarity rating of 3.58 and only small effects of the particular pair of good-poor patterns.

Interstimulus similarity. With the sequential "samedifferent" procedure, on each different trial a particular pair of stimuli is involved, and the interstimulus similarity of this pair might influence RT. The good-good different stimuli were faster than the poor-poor different stimuli, and this is what would be expected on the basis of interstimulus similarity. However, the good-poor and poor-good stimulus conditions should have been the fastest because these stimuli are most dissimilar, but they were the slowest when the poor stimulus was the target and intermediate between the good-good and the poor-poor conditions when the good stimulus was the target. Thus, somewhat surprisingly, there was no clear effect of interstimulus similarity on the different RT.

Similarity space. Goodness is correlated with isolation of stimuli in a similarity space, because the two poor 
patterns are more similar to each other than are the two good patterns. Thus the two good patterns are more isolated both from each other and from the poor patterns than the two poor patterns are from each other or from the good patterns. This isolation could account for the faster "same" responses to good patterns than to poor patterns. It might also account for the fact that good-poor RTs were faster than poor-good RTs, on the assumption that the effect of the relative isolation is greater in memory than when encoding is occurring. It might further account for the faster responding to different good patterns than to different poor patterns, but in this case, goodness, interstimulus similarity, and isolation in similarity space all predict the same result.

In conclusion, the two major findings of Experiment 1 are that "same" responses to good patterns are faster than those to poor patterns and that "different" responses are faster when a good pattern is the target held in memory than when a poor pattern is the target. Since these two results are at least consonant with the idea that good stimuli are isolated in a similarity space, with its resultant faster processing, an additional experiment was carried out to provide further clarification of the issues.

\section{EXPERIMENT 2: TWO STIMULUS ALTERNATIVES}

Because the results of Experiment 1 showed effects that could be due to a similarity space, in Experiment 2 we used blocks of trials in which only two stimuli could occur. This procedure minimized the possibility that the more complex similarity space was used by having a single interstimulus similarity operate for each block of trials.

Although only two stimuli could occur in a single block of trials, all six pairs of the four stimuli of Experiment 1 were used in different blocks. This procedure makes it possible to compare the speed of both the "same" and the "different" responses as a function of the particular alternative stimulus, and thus of the particular interstimulus similarity. If the results show no consistent effects of interstimulus similarity (as in Experiment 1) but do show effects of stimulus goodness, the effect of goodness can be more certainly ascribed to its stimulus-specific properties.

\section{Method}

Subjects. Twelve Yale University students, eight male and four female, each received course credit, pay, or a combination of both to participate in this experiment for a total of approximately $2.5 \mathrm{~h}$. Three of the subjects had participated in Experiment 1 .

Stimuli. The patterns of Experiment 1 were used, but the maximum vertical or horizontal visual angle of the projected image was reduced to approximately $1.0 \mathrm{deg}$. Both the target and display items were presented in the center of the viewing field.

Procedure. The task was again a sequential "same-different" identification. In each block, only two of the four stimuli could appear. On each trial, one of the two stimuli was presented as the first (target) item for $1.0 \mathrm{sec}$, followed by a 1.0 -sec delay. One of the same two stimuli was then presented as the second (display) item and remained on until a response was made. Between trials, there was a delay of $3.25 \mathrm{sec}$, during which slides were changed and responses and RTs were recorded. Timing was controlled by logic circuitry. Responses and RT (to the nearest millisecond) were recorded by a computer, a fact that permitted the reduced intertrial interval. Responses were made in the same manner as in Experiment 1. Response assignment was quasirandom: Six subjects (four male and two female) were verbally instructed to press the right-hand key if the stimuli were the same and the lefthand key if the stimuli were different; the remaining six subjects (four male and two female) were given the reverse response assignment.

Before each block, subjects were shown exemplars of the two stimuli for that block. They were instructed to respond as rapidly as possible while trying to avoid errors.

Experimental design. Each subject participated in two sessions in a single day. Each session lasted approximately $1 \mathrm{~h}$ and $15 \mathrm{~min}$ and consisted of six blocks of trials. The subject was given a short break between blocks and a longer break between sessions.

Each block consisted of a total of 48 trials, half of which were same pairs (12 trials of each of the two same pairs of stimuli), and half of which were different pairs (12 trials of each of the two different pairs of stimuli). Thus, within a block, each response was required on half of the trials.

In each session, every stimulus occurred in three blocks (paired once with each of the other three stimuli); each same pair therefore occurred in three blocks, whereas each different pair occurred in only one block. As a consequence, each same pair occurred three times as often as each different pair over the course of the experiment.

The order in which the pairs of stimuli were assigned to blocks was balanced across subjects in a Latin square design for each group of six subjects. The order of blocks was reversed for each subject in the second session. The pairs of stimuli within a block were presented in a different quasirandom order for each block, with the following constraints: No pairing could occur more than five times sequentially, and in order to prevent the subject from trying to guess the stimulus order, sequences that appeared to the experimenter to have some detectable pattern were not used.

Analyses. Each block provided a total of 12 trials per subject for each pair. Error trials were eliminated, and a median RT was calculated for each of the four stimulus pairs in each of the blocks for each session. These medians provided the basis for all further RT analyses. Statements of significance are at $p<.05$ and are based on analyses of variance with Newman-Keuls tests for pairwise comparisons.

Mean error rates for each condition ranged from $2 \%$ to $5 \%$ and were positively correlated with mean RT. No further analyses of errors were made.

\section{Results and Discussion}

Analyses were collapsed across sex and hand. Although there was a significant decrease in overall RT across sessions, this change did not interact with any principal comparison. Therefore, the mean values for the various conditions are presented in Table 2 , collapsed across sessions.

Effects of conditions. Overall mean RT to same pairs was faster than that for different pairs. Once again, however, this difference is not of primary importance for the issues investigated in this experiment, so no specific interpretation is given. Of interest are the differences between good and poor stimuli within each response type. 
Table 2

Mean Reaction Time (in Milliseconds) for Each Stimulus Pairing in Experiment 2: Two Stimulus Alternatives

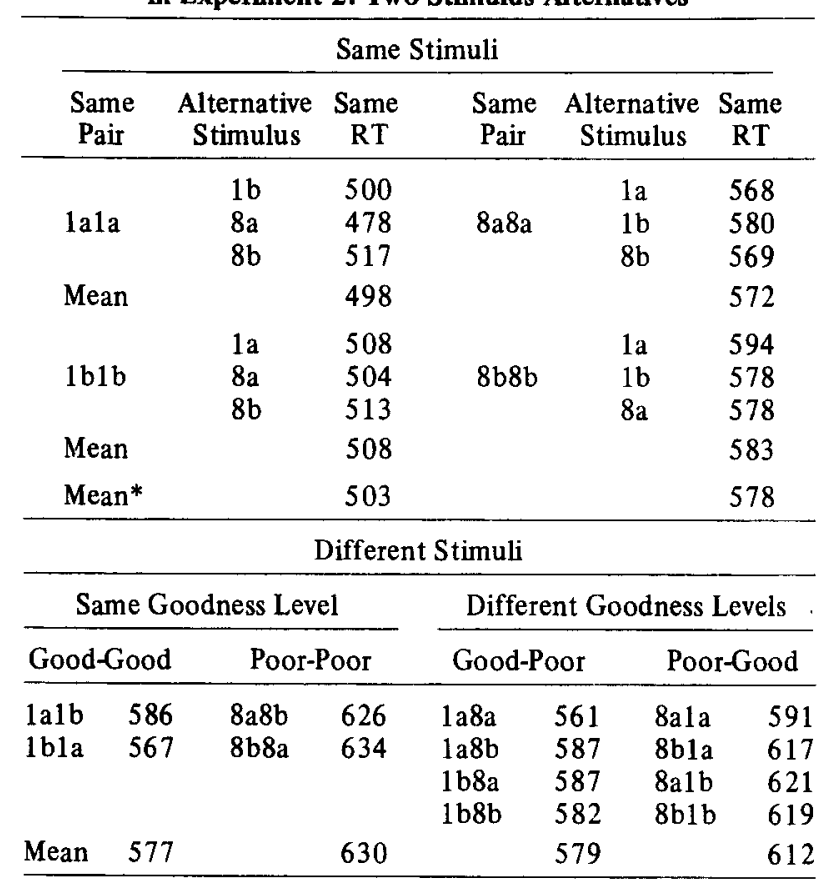

Note-Means for each pairing are collapsed across blocks, sessions, and subjects. *Overall mean.

Same stimuli. Each stimulus was paired with each of the other three stimuli in separate blocks. The three mean RTs for each same pair are presented in the upper portion of Table 2. The alternative stimulus present in each block is indicated next to the RT.

There were significant differences between the goodgood and the poor-poor "same" responses, with an overall mean difference of $75 \mathrm{msec}$. In contrast, the mean difference between stimuli within a goodness level was $10 \mathrm{msec}$ for the good patterns and $11 \mathrm{msec}$ for the poor patterns. This result replicates that of Experiment 1 , with "same" responses to good patterns being considerably faster than "same" responses to poor patterns.

Further analysis can clarify the relative importance of goodness and interstimulus similarity in determining the speed of the "same" response. Consider first the extent to which the "same" RT to stimuli of one goodness level depends on the goodness level of the alternative stimulus. The two good stimuli ( $1 \mathrm{a}$ and $1 \mathrm{~b}$ ), when paired with each other, show a mean RT of $504 \mathrm{msec}$, and they differ from each other by only $8 \mathrm{msec}$. These same two good stimuli, when paired with the poor stimuli ( $8 \mathrm{a}$ and $8 \mathrm{~b}$ ), show a mean RT of $503 \mathrm{msec}$, with a range of from 478 to 517 msec. Thus the "same" RT to a good stimulus is unaffected by the stimulus alternative with which it is paired. In a similar fashion, the two poor stimuli, when paired with each other, show a mean RT of $574 \mathrm{msec}$, differing from each other only by $9 \mathrm{msec}$. These same two poor stimuli, when paired with the good stimuli, show a mean RT of $580 \mathrm{msec}$, with a range of from 568 to $594 \mathrm{msec}$.

In brief, these data for "same" RTs indicate that the RT to a particular stimulus is relatively unaffected by the stimulus alternative or by the interstimulus similarity between the two stimuli in a block, but it is greatly affected by the goodness level of the specific stimulus.

A corollary to this conclusion is the fact that the similarity between a pair of stimuli does not act in a symmetric fashion to determine the speed of the "same" response, since when the goodness levels of the two stimuli in a pair differ, so also do the "same" RTs. Thus we have essentially no evidence that interstimulus similarity is operative in determining these "same" RTs, but considerable evidence that the goodness level of the specific stimulus is.

Different stimuli, same goodness level. The lower left section of Table 2 indicates a statistically significant mean difference of $53 \mathrm{msec}$ between good-good and poor-poor stimulus pairs, as contrasted to differences within goodness levels, which were a nonsignificant $19 \mathrm{msec}$ for good stimuli and $8 \mathrm{msec}$ for poor stimuli. This result again replicates that found in Experiment 1 : Good stimuli are responded to more rapidly than are poor stimuli when the stimuli are different but of the same goodness level.

Different stimuli, different goodness levels. The RTs for the eight pairs of stimuli in which one good stimulus is paired with one poor stimulus are shown in the lower right section of Table 2. For each of the four stimulus pairings, RT was faster when the good stimulus was the target than when the pairing was reversed so that the poor stimulus was the target. The differences ranged from 30 to $37 \mathrm{msec}$, with a mean difference of $33 \mathrm{msec}$.

Effects of target and display. The mean RT for all different stimuli with a good target was $578 \mathrm{msec}$, whereas that for poor targets was $618 \mathrm{msec}$; this is a significant difference of $40 \mathrm{msec}$. In contrast, there was no significant difference between the combined good display $(600 \mathrm{msec})$ and the combined poor display $(596 \mathrm{msec})$. As in Experiment 1, this result can be interpreted as indicating that the primary effect of stimulus goodness is in the processing of the memory item.

Effect of similarity with different stimuli. It has already been noted that interstimulus similarity has no effect on the speed of the "same" response, although goodness has a large effect. The same conclusion can be drawn with respect to the effect of interstimulus similarity on the speed of the "different" response. The difference in RT for the two conditions involving different stimuli at the same goodness level could by itself be interpreted as a result of interstimulus similarity, since the more similar poor patterns were discriminated more slowly than the less similar good patterns. However, as in Experiment 1, further comparisons with the RTs for the different stimuli with different goodness levels do not allow this interpretation. If interstimulus similarity were the determining factor of RT, the good-poor and poor-good pairings should have 
provided the fastest RTs. In contrast, RTs for the good-poor and poor-good conditions were intermediate between those for good-good stimuli and poor-poor stimuli. Still further, the substantial difference in RT when a good rather than a poor stimulus pattern was the target or memory item suggests that interstimulus similarity is a weak factor at best in determining the RT to different stimuli.

\section{SUMMARY AND GENERAL DISCUSSION}

The experiments reported here attempted to clarify the effect of goodness on information processing by the use of a sequential "same-different" task, allowing a differentiation of the role of goodness in memory and in encoding.

\section{Memory and Encoding}

The sequential "same-different" task shows a substantial effect of pattern goodness on processing. RT to two configurally good stimuli is faster than that to two poor stimuli, both for stimuli that are the same and for stimuli that are different but at the same goodness level. In these two conditions, however, both the target item presumably held in memory and the display item that presumably must be encoded have the same goodness. There is, therefore, complete confounding between the roles of goodness in the target and in the display item, so that no conclusions can be drawn that differentiate between the role of memory and encoding.

Such differentiation is possible, however, in the third condition, when the target and display items have different goodness. In both experiments, there was a clear effect of the target item but not of the display item on RTs. This result alone suggests that the primary effect of pattern goodness occurs in the processing of the memory item. However, it should be noted that the amount of difference in RT between simple reversal of target and display stimuli is never as great as that obtained when both target and display belong to the same goodness level. Insofar as we can distinguish between memory and encoding effects, our data show that memory is the more important factor. It is not possible, however, to eliminate the role of encoding entirely, and the role of encoding may well be interactive with the role of memory in this task.

Are other results compatible with those obtained here? The data of both Checkosky and Whitlock (1973) and Pomerantz (1977) were reanalyzed in terms of the conditions used in our experiments, and in both cases the good-good "sames" were faster than poor-poor "sames," good-good "differents" were faster than poor-poor "differents," and good-poor "differents" were faster than poor-good "differents." Both of these papers had been interpreted as showing a primary effect of memory on the processing of goodness, but the specific agreement in this pattern of results is quite striking in light of the differences in procedures.
Whereas other studies have been interpreted as showing encoding effects, it is possible to account for the principal results of those studies in terms of memory effects. Following Pomerantz (1977), it is reasonable to think of the two-choice discrimination task used by Garner and Sutliff (1974) as a "same-different" task in which the subject specifies the target item. Assume that the subject in fact handles the two-choice task by first fixing one item in memory and then using a go/no-go strategy, so that the subject is essentially deciding whether the immediately displayed stimulus is or is not the item in memory. If a good item is easier to store or focus than a poor item, the subject will tend to use good patterns as targets, and, consequently, good stimuli will be responded to faster than poor stimuli. Garner and Sutliff obtained just this result.

Ruth (1976) argued for an effect of goodness on encoding, basing her conclusions largely on the facts that unexpected good stimuli were processed faster than unexpected poor ones and that the difference between good expected and unexpected stimuli was less than that between poor expected and unexpected stimuli. These results, however, can be accommodated by assuming that the effectiveness of an expectancy is greater for good than for poor patterns and that good patterns always are expected to some extent, regardless of the particular expectancy induced experimentally.

It is difficult to accommodate the results of Bell and Handel (1976) to a predominant effect of memory, but there is little reason to assume that goodness has no effect on encoding. Our conclusion is simply that insofar as the effects of memory and encoding have been separated by the use of the sequential task, the effect of goodness is greater on the item held in memory than it is on the item displayed for comparison.

\section{The Role of Similarity}

The effects of interstimulus similarity on RT in tasks involving choice or comparison of stimuli are so well documented that RT is frequently used as a measure of interstimulus similarity. Despite this fact, in the present experiments, the stimulus-specific property of goodness clearly dominated the results. Whereas the isolation of particular stimuli in a similarity space was a possible explanation for the results of Experiment 1, in which four alternative stimuli were used, its role as a simple explanatory concept was eliminated by the use of just two stimulus alternatives in a block of trials in Experiment 2 . In addition, it was shown that interstimulus similarity did not provide a straightforward account of goodness effects: "same" RTs did not vary with the alternative item, and good-poor "differents" were faster than poor-good "differents."

However, these results do not completely invalidate the role of similarity as an explanatory construct in understanding goodness. Goodness and similarity are both highly complex concepts, and it is therefore difficult to relate them in a simple way. Our results do, 
however, place constraints on the concepts of similarity that are compatible with a description of the effect of goodness.

Several recent studies have provided analyses of similarity that are consistent with some of the results described here. Podgorny and Garner (1979) showed that "same" RTs for matching of letters could be analyzed in terms of differential intraobject similarity or self-similarity. They argued that faster RTs indicated greater intraobject similarity. The results we have described would thus be consistent with the notion that good patterns have higher intraobject similarity.

In addition, several authors have argued that similarity can accommodate asymmetries. Tversky (1977) argued for a feature-matching model in which the order of item presentation affected judged similarity. Krumhansl (1978) elaborated a distance-density model that reconciled asymmetries to a geometric model of similarity. Again, both of these positions would be consistent with an interpretation of goodness as reflecting the influence of an asymmetric similarity.

There are thus several approaches to similarity that do address some of the issues we have raised about goodness. However, whereas most conceptions of similarity are dependent on taking into account the stimuli presented in the experimental set, our results have demonstrated that the similarity relations of the experimental set are not adequate to explain the role of goodness.

But the fact that similarity relations among items in the explicit set do not account for the data is not equivalent to a rejection of the role of similarity. As Garner (1962) originally argued, the notion of goodness is closely tied to that of an inferred set. The goodness of a particular stimulus is due to the number of inferred alternatives rather than to the number of actual alternative stimuli; good patterns have few inferred alternatives or equivalent stimuli. Such a notion suggests that good patterns can be thought of as isolated in an inferred similarity space. Thus goodness entails certain relations in that space, and goodness and the inferred space are intimately related.

In summary, our results do not suggest an incompatibility of goodness and similarity explanations. Rather, we have demonstrated stimulus-specific effects of goodness insofar as neither explicit interstimulus similarity nor similarity space accounts for our results.
However, to the extent that goodness is describable in terms of inferred alternatives, it is quite compatible with a description of implicit similarity relations of specific stimuli.

\section{REFERENCES}

Bell, H. H., \& Handel, S. The role of pattern goodness in the reproduction of backward masked patterns. Journal of Experimental Psychology: Human Perception and Performance, 1976, 2, 139-150.

Checkosky, S. F., \& Whitlock, D. The effects of pattern goodness on recognition time in a memory search task. Journal of Experimental Psychology, 1973, 100, 341-348.

Clement, D. E., \& Varnadoe, K. W. Pattern uncertainty and the discrimination of visual patterns. Perception \& Psychophysics, 1967, 2, 427-431.

GARNER, W. R. Uncertainty and structure as psychological concepts. New York: Wiley, 1962.

GARNER, W. R. The processing of information and structure. Potomac, Md: Erlbaum, 1974.

Garner, W. R., \& Clement, D. E. Goodness of pattern and pattern uncertainty. Journal of Verbal Learning and Verbal Behavior, 1963, 2, 446-452.

Garner, W. R., \& Sutliff, D. The effects of goodness on encoding time in visual pattern discrimination. Perception \& Psychophysics, 1974, 16, 426-430.

King, M. C., Crist, W. B., \& Lockhead, G. R. Context and goodness in a focusing task. Perception \& Psychophysics, 1979, 26, 305-311.

KrumhansL, C. L. Concerning the applicability of geometric models to similarity data: The interrelationship between similarity and spatial density. Psychological Review, 1978, 85, 455-463.

Lockнe ad, G. R. Processing dimensional stimuli: A note. Psychological Review, 1972, 79, 410-419.

Lockнеad, G. R., \& King, M. C. Classifying integral stimuli. Journal of Experimental Psychology: Human Perception and Performance, 1977, 3, 436-443.

Podgorny, P., \& Garner, W. R. Reaction time as a measure of inter- and intraobject visual similarity: Letters of the alphabet. Perception \& Psychophysics, 1979, 26, 37-52.

Pomerantz, J. R. Pattern goodness and speed of encoding. Memory \& Cognition, 1977, 5, 235-241.

Ruth, D. S. The effects of pattern goodness on automatic and strategy dependent processes. Unpublished doctoral dissertation, Yale University, 1976.

STERnberg, S. Two operations in character recognition: Some evidence from reaction-time measurements. Perception \& Psychophysics, 1967, 2, 45-53.

Tve rsky, A. Features of similarity. Psychological Review, 1977, 84, 327-352.

(Accepted for publication November 10, 1980.) 